Z. Klin. Chem. Klin. Biochem.

13. Jg. 1975 , S. $231-237$

\title{
Gaschromatographische Bestimmung von Lipiden in Blut, Plasma oder in Organgeweben
}

\author{
Von M. Radke, H. Schäfer und J. D. Kruse-Jarres \\ Aus der Chirurgischen Universitätsklinik (Direktor Prof. Dr. M. Schwaiger) Freiburg im Breisgau
}

(Eingegangen am 15. Oktober 1974/25. März 1975)

Es wird eine Methode zur Bestimmung von Cholesterinestern, Triglyceriden und Lecithin angegeben. Sie umfaßt die Extraktion der Lipide, die dünnschichtchromatographische Auftrennung sowie die quantitative gaschromatographische Analyse der veresterten Fettsäuren nach der Umesterung zu den 2-Methoxyäthylestern. Das Derivatisierungsverfahren ist für kurz- und langkettige freie und veresterte Fettsäuren geeignet.

\section{Quantitation of lipids in blood, plasma, and tissues by gas-liquid chromatography}

A method is described for the determination of cholesteryl esters, triglycerides, and lecithin. The lipids are extracted, separated by thin-layer chromatography, and quantitated by gas chromatography following the esterification of the fatty acids to their 2-methoxyethyl esters. A transesterification procedure is described, which is suitable for short- and long-chain free and esterified fatty acids.

Für die Analyse der Lipide hat sich die Gaschromatographie als ein wertvolles Hilfsmittel erwiesen. Eine Reihe von Autoren hat über die gaschromatographische Bestimmung der Lipide im Zusammenhang mit klinischchemischen Fragestellungen berichtet, vornehmlich über die Ermittlung von Fettsäuremustern einzelner Lipidklassen (1-5). Hierfür ist zunächst eine Auftrennung des Lipidgemisches erforderlich. Die Säulenchromatographie wurde weitgehend durch die dünnschichtchromạtographische Trennung abgelöst, weil mii dieser Methode bei geringerem Zeitaufwand eine bessere Trennung gelingt. Auf die Trennung folgt die Umesterung der Lipide mit einem Alkohol, meist Methanol, in Gegenwart saurer oder basischer Katalysatoren, bei der die mit Glycerin oder Cholesterin veresterten Fettsäuren in die Ester des betreffenden Alkohols übergeführt werden. Die Methylester der Carbonsäuren mit etwa zwölf bis vierundzwanzig Kohlenstoffatomen sind für die quantitative Analyse gut geeignet. Ester mit geringerer Kettenlänge werden ohne besondere Vorkehrungen zur Vermeidung von Verdampfungsverlusten nicht vollständig erfaßt $(6,7,8)$.

Bei einer Diskussion der posttraumatischen Fettembolie hat Stolz (9) auf die Möglichkeit hingewiesen, die Entstehung respiratorischer Störungen auf das vermehrte Angebot kurzkettiger Fettsäuren in der Lunge zurückzuführen. Heller \& Durst (10) erwähnen gaschromatographische Untersuchungen der Serumlipide, nach denen sich im Schock die langkettigen Fettsäuren vermehrt nachweisen lassen, während die kurzkettigen praktisch verschwinden. Da wir im Zusammenhang mit Untersuchungen zur Fettembolie an einer Klärung dieser Fragen interessiert waren, haben wir.eine Methode ausgearbeitet, mit der auch kurzkettige freie und veresterte Fettsäuren sicher erfaßt werden.

\section{Material und Methoden}

\section{Lösungsmittel}

Chloroform, Methanol, Petroläther $\left(\mathrm{Kp} 40-60^{\circ} \mathrm{C}\right)$, Diäthyläther stabilisiert mit 2,6-Di-tert.-butyl-4-methylphenol (BHT), n-Hexan, Toluol, p.a. (E. MERCK).

\section{Reagenzien}

Bortrifluorid-Diäthyläther-Komplex, Hydrochinon, zur Synthese, Platin[IV]-oxid, Hydrierkatalysator (MERCK-SCHUCHARDT); 2-Methoxyäthanol, Natriumchlorid, Natriumhydrogencarbonat, p.a., $2^{\prime} .7^{\prime}$-Dichlorfluorescein $0,1 \%$, Sprühreagenz (E. MERCK).

\section{Hilfsmittel}

Faltenfilter Nr. 589/3 1/2 (SCHLEICHER \& SCHÜLL); Molekularsieb 5 A, Stäbchen (HEWLETT-RACKARD).

\section{Fertigplatten für die Dünnschichtchromatographie}

Kieselgel 60, $50 \times 200 \mathrm{~mm}$ - Schichtdicke 0,25 mm, $200 \times$ $200 \mathrm{~mm}$ - Schichtdicke 0,5 mm (E. MERCK); SILGUR-25 $\mathrm{UV}_{254}, 200 \times 200 \mathrm{~mm}$ (MACHEREY NAGEL \& Co).

Stationäre Phasen für die Gaschromatographie

SILAR-10C (neuere Bezeichnung: APOLAR-10C), OV 275, GAS-CHROM Q 80-100 mesh (APPLIED SCIENCE Laboratories); $100 \mathrm{~g}$ Apiezon $\mathrm{L}$ auf $1 \mathrm{~kg}$ 80-100 mesh Chromosorb W-AW-DMCS (HEWLETT-PACKARD). 


\section{Bezugssubstanzen für die Gaschromatographie}

3:0 - 5:0-Fettsäuren, zur Synthese (MERCK-SCHUCHARDT); 6:0 - 10:0-Fettsäuren, puriss. (CARL ROTH); Pentadecansäure, Tricaprin puriss. (FLUKA); 6:0 - 18:0, 18:1, 18:2, 18:3 -Fettsäuremethylester, Bezugssubstanzen für die Gaschromatographie (E. MERCK); n-5,8,11,14-Eicosatetraensäuremethylester, $n-4,7,10,13,16,19$-Docosahexaensäuremethylester (WERNER GUUNTHER); Fettsäuren: Kit ES, EU; Methylester: Kit OS, Mixture K 107, L 209; Äthylester: Kit ES; n-8,11,14Eicosatriensäure; Triglyceride: Kit EOS (APPLIED SCIENCE Laboratories); 10:0 - 18:0, 18:1 -Fettsäurecholesterinester, für biochemische Zwecke bzw. Licristal (E. MERCK); Lecithin aus Eiern, reinst (E. MERCK) wurde nach dünnschichtchromatographischer Reinigung verwendet. 2-Methoxyäthylester: 3:0 - 10:0-Fettsäure-2-methoxyäthylester wurden aus den Säurechloriden nach L.c. (11) dargestellt; Reinheit (durch GC) nach Vakuumdestillation: über 99\%; 15:0, 18:0, 18:1, 18:2, $18: 3,20: 4,22: 6$-Fettsäure-2-methoxyäthylester wurden in Mengen von 0,2-0,6 mmol aus den Methylestern nach der unten angegebenen Methode dargestellt; Reinheit (durch GC) nach dünnschichtchromatographischer Reinigung: über $98 \%$.

\section{Lipidextraktion}

\section{Allgemeine Hinweise}

Alle Extraktionsschritte werden unter Stickstoff durchgefuihrt, den man über eine Säule mit Molekularsieb leitet. Es werden Scheidetrichter mit P.T.F.E.-Küken verwendet. Chloroform wird mit $1 \mathrm{mmol} / 1$ Hydrochinon als Antioxydans versetzt. Das Eindampfen der Lipidlösungen erfolgt im Vakuum (Rotationsverdampfer) bei $30^{\circ} \mathrm{C}$, die Aufbewahrung bei $-30^{\circ} \mathrm{C}$ unter Stickstoff.

\section{Extraktion von Blut und Plasma nach Farquhar (12) modifiziert:} $4 \mathrm{ml}$ Blut läßt man in ein Polyäthylenröhrchen fließen, dessen Innenwand mit EDTA (Dinatriumsalz der Äthylendiamintetraessigsäure) präpariert ist. Man bestimmt den Hämatokrit mit einer Mikro-Hämatokrit-Zentrifuge. $1 \mathrm{ml}$ Blut werden abpipettiert und tropfenweise unter Umschwenken $\mathrm{zu} 8 \mathrm{ml}$ Methanol gegeben. Man läßt 10 min im Dunkeln stehen und fügt $2 \mathrm{ml}$ einer Lösung von $45 \mu \mathrm{mol}$ Tricaprin und $111 \mu \mathrm{mol}$ Cholesterincaprinat in $100 \mathrm{ml}$ Chloroform.zu. Anschließend werden $10 \mathrm{ml}$ Chloroform zugegeben. Nach Zerkleinern der Klumpen läßt man 30 min im Dunkeln stehen. Man fügt $4 \mathrm{ml}$ Chloroform zu und filtriert über ein Faltenfilter in einen $25 \mathrm{ml}$ Meßzylinder. $20 \mathrm{ml}$ Filtrat werden aufgefangen und im Scheidetrichter mit $4 \mathrm{ml}$ wäßr. Natriumchloridlösung $0,1 \mathrm{~mol} / 1$ geschüttelt. Die untere Phase wird abgetrennt und zweimal mit je $5 \mathrm{ml} \mathrm{Methanol/wäßr.} \mathrm{Natriumchloridlösung} 0,1 \mathrm{~mol} / 1$ Chloroform $(470 \mathrm{ml}+480 \mathrm{ml}+30 \mathrm{ml})$ ausgeschüttelt. Der gereinigte Extrakt wird bei $-30^{\circ} \mathrm{C}$ aufbewahrt. Zur Gewinnung von Plasma werden $3 \mathrm{ml}$ Blut $20 \mathrm{~min}$ bei $2000 \mathrm{~g}$ zentrifugiert. Die Extraktion von $1 \mathrm{ml}$ Plasma erfolgt in gleicher Weise wie beim Blut.

Die Organextraktionen werden nach Folch et al. (13) durchgeführt.

\section{Dünnschichtchromatographie}

\section{Allgemeine Hinweise}

Vor Einbringen des Laufmittels werden die Dünnschichtchromatographie-Tanks mit Stickstoff gespült. Das Laufmittel ist täglich zu erneuern. Für Extrakte aus Blut und Plasma werden Kieselgel 60-Platten $(50 \times 200 \mathrm{~mm})$, für Organextrakte SILGURPlatten verwendet. Diese besitzen eine Auftragzone aus Kieselgur, die das rasche Aufbringen größerer Lösungsmengen erleichtert. Unmittelbar vor dem Gebrauch werden die Platten eine Stunde bei $120^{\circ} \mathrm{C}$ aktiviert. Die Probe wird mit einer $10 \mu l$ Hamilton-Spritze im Stickstoffstrom strichformig aufgetragen. Nach der Entwicklung läßt man 2', 7'-Dichlorfluorescein einwirken und markiert die Banden unter UV-Licht (366 nm).

\section{Trennung der polaren Lipide}

Man entwickelt nach (14) mit Chloroform/Methanol/Wasser/ Eisessig $(65 \mathrm{ml}+25 \mathrm{ml}+4 \mathrm{ml}+1 \mathrm{ml})$. Nach steigendem $R_{F}$ Wert können folgende Banden zugeordnet werden: Lysolecithin,
Sphingomyelin, Lecithin, Kephalin und Cholesterin. Freie Fettsäuren, Triglyceride und Cholesterinester bilden eine einzige Zone nahe der Lösungsmittelfront. Sie wird gemeinsam mit der Cholesterin-Zone in eine Chromatographiesäule $(15 \times 1 \mathrm{~cm})$ übergeführt und mit $25 \mathrm{ml}$ Chloroform eluiert.

\section{Trennung der unpolaren Lipide}

Es wird nach 1.c. (7) mit Petroläther/Diäthyläther $(90 \mathrm{ml}+10 \mathrm{ml})$ entwickelt. Nach steigendem $R_{F}$-Wert können folgende Banden zugeordnet werden: Cholesterin, freie Fettsäuren, Trigly ceride und Cholesterinester.

\section{Umesterung}

\section{Allgemeine Hinweise}

Die Umesterung wird unter Stickstoff in $10 \mathrm{ml}$. Reagenzgläsern mit Schraubgewinde (S.V.L., SOVIREL) durchgeführt. Mit Schraubklappe und Dichtungsring wird ein Steigrohr $200 \times 8 \mathrm{~mm}$ aufgesetzt, das am oberen Ende eine mit Glaswolle fixierte $30 \mathrm{~mm}$ lange Schicht Molekularsieb enthält. $\mathrm{Da}$ bei der Reaktion Äther entweicht, arbeitet man unter dem Abzug. Der Bortrifluorid-Diäthyläther-Komplex wird bei $0^{\circ} \mathrm{C}$ gelagert. Das reine Reagenz ist farblos; ist es gelblich verfärbt, wird es unter Feuchtigkeitsausschluß über eine $30 \mathrm{~cm}$ Vigreux-Kolonne destilliert (Kp. $\left.126^{\circ} \mathrm{C}\right)$.

\section{Polare Lipide}

Die Kieselgelschicht, die das polare Lipid (Lecithin oder Kephalin) enthält, wird in ein $10 \mathrm{ml}$ Reagenzglas (s.o.) gebracht. Man fügt $100 \mu$ Pentadecansäure-Standardlösung $(0,825 \mathrm{mmol}$ Pentadecansäure in $100 \mathrm{ml}$ 2-Methoxyäthanol), $3 \mathrm{ml} \mathrm{2-Methoxy-}$ äthanol und $1 \mathrm{ml}$ Bortrifluorid-Diäthyäther-Komplex zu. Nach Zugabe einiger Stäbchen Molekularsieb setzt man das Steigrohr auf und erwärmt im Paraffinbad $20 \mathrm{~min}$ auf $110^{\circ} \mathrm{C}$. Man kühlt auf $0^{\circ} \mathrm{C}$, fügt $5 \mathrm{ml}$ destilliertes Wasser zu, schüttelt kräftig und läßt die Suspension in $7 \mathrm{ml}$ gesättigte wäßr. Natriumhydrogencarbonatlösung einfließen. Anschließend schüttelt man mit $10 \mathrm{ml}$ Petroläther aus. Die Oberphase wird abgetrennt und zur vollständigen Entfernung des suspendierten Kieselgels zwei- bis dreimal mit $3 \mathrm{ml}$ wäßr. Natriumchloridlösung $0,1 \mathrm{~mol} / 1 \mathrm{ge}-$ waschen. Man vereinigt die Unterphasen und wiederholt die Extraktion. Die vereinigten Oberphasen werden auf $0,5 \mathrm{ml}$ eingeengt.

\section{Unpolare Lipide}

Vor der Umesterung werden die dünnschichtchromatographisch getrennten Lipide aus dem Kieselgel eluiert. Dazu wird die Kieselgelschicht, die das unpolare Lipid (Triglyceride oder Cholesterinester) enthält, in ein $10 \mathrm{ml}$ Reagenzglas (s. o.) mit Schraubverschluß gebracht, das Petroläther/Diäthyläther $(4 \mathrm{ml}+1 \mathrm{ml})$ enthält. Es wird geschüttelt und anschließend zentrifugiert. Man wiederholt die Extraktion zweimal. Die vereinigten Extrakte werden eingedampft. Man nimmt den Rückstand in $0,5 \mathrm{ml}$ Toluol auf und bringt ihn in ein $10 \mathrm{ml}$ Reagenzglas (s.o.). Nach Zugabe von 2,5 ml 2-Methoxyäthanol, $1 \mathrm{ml}$ Bor trifluorid-Diäthyläther-Komplex sowie einigen Stäbchen Molekularsieb setzt man das Steigrohr auf und erwärmt im Paraffinbad 20 min auf $110^{\circ} \mathrm{C}$. Man kühlt auf $0^{\circ} \mathrm{C}$, fügt $5 \mathrm{ml}$ destilliertes Wasser hinzu, schüttelt und läßt in $7 \mathrm{ml}$ gesättigte wäßr. Natriumhydrogencarbonatlösung einfließen. Es wird mit viermal $5 \mathrm{ml}$ Petroläther extrahiert. Die vereinigten Extrạkte werden auf $50 \mu \mathrm{l}$ eingedampft. Zur Reinigung der Ester wird die toluolhaltige Lösung unter dem Abzug auf die Dünnschichtchromatographie-Platte $(50 \times 200 \mathrm{~mm})$ aufgetragen. Man entwickelt mit Petroläther/Diäthyläther $(80 \mathrm{ml}+20 \mathrm{ml})$. Nach der Einwirkung von 2', $7^{\prime}$-Dichlorfluorescein wird die Esterżone unter UV-Licht (366 nm) markiert. Sie wird abgekratzt und zweimal mit Petroläther/Diäthyläther $(4 \mathrm{ml}+1 \mathrm{ml})$ extrahiert. Die vereinigten Extrakte werden auf $50 \mu$ l eingedampft.

\section{Gaschromatographie}

\section{Allgemeine Hinweise}

Es wurde ein Gaschromatograph 7620A mit Integrator 3370B und automatischer Einspritzvorrichtung 7670A der Firma HEWLETT PACKARD verwendet. Durch den Einsatz eines 
Tab. 1. Umesterung von Methylestern, Triglyceriden und Cholesterinestern zu den 2-Methoxyäthylestern

\begin{tabular}{|c|c|c|c|c|c|c|c|c|c|}
\hline \multicolumn{4}{|c|}{$\begin{array}{l}\left.\text { Methylester }(n=10)^{1}\right) \\
\text { Massefraktionen } \cdot 10^{2}\end{array}$} & \multicolumn{3}{|c|}{$\begin{array}{l}\text { Triglyceride }(n=5) \\
\text { Massefraktionen } \cdot 10^{2}\end{array}$} & \multicolumn{3}{|c|}{$\begin{array}{l}\text { Cholesterinester }(n=5) \\
\text { Massefraktionen } \cdot 10^{2}\end{array}$} \\
\hline $\mathrm{FS}^{2}$ ) & $\operatorname{Ber}(x)$ & $\operatorname{Gef}(\bar{x})$ & $\pm S$ & $\operatorname{Ber}(x)$ & $\operatorname{Gef}(\bar{x})$ & $\pm s$ & $\left.\operatorname{Ber}(x)^{3}\right)$ & $\operatorname{Gef}(\bar{x})$ & $\pm s$ \\
\hline $6: 0$ & 10,55 & 10,54 & 0,19 & 11,37 & 10,04 & 0,67 & - & - & - \\
\hline $7: 0$ & 3,81 & 3,79 & 0,07 & - & - & - & - & - & - \\
\hline $8: 0$ & 9,89 & 9,69 & 0,17 & 35,64 & 36,01 & 0,49 & - & - & - \\
\hline $9: 0$ & 4,23 & 4,08 & 0,07 & - & - & - & - & - & - \\
\hline $10: 0$ & 10,17 & 10,43 & 0,12 & 14,64 & 15,21 & 0,23 & 8,83 & 8,93 & 0,13 \\
\hline $11: 0$ & 4,58 & 4,67 & 0,02 & - & - & - & - & _ & - \\
\hline $12: 0$ & 10,22 & 10,32 & 0,04 & 7,84 & 8,33 & 0,20 & 24,15 & 25,39 & 0,17 \\
\hline $13: 0$ & 1,95 & 2,17 & 0,03 & - & - & - & - & - & - \\
\hline $14: 0$ & 10,78 & 10,77 & 0,10 & 5,06 & 5,43 & 0,13 & 11,54 & 12,33 & 0,09 \\
\hline $15: 0$ & 4,28 & 4,53 & 0,06 & - & - & - & - & - & - \\
\hline $16: 0$ & 10,01 & 10,08 & 0,16 & 5,25 & 5,38 & 0,20 & 20,60 & 21,09 & 0,27 \\
\hline $17: 0$ & 0,99 & 1,11 & 0,02 & 2,74 & 2,74 & 0,04 & - & - & - \\
\hline 18:0 & 8,08 & 8,08 & 0,09 & 6,01 & 6,04 & 0,10 & 12,69 & 13,07 & 0,26 \\
\hline $18: 1$ & 10,46 & 9,74 & 0,16 & - & - & - & 22,19 & 19,19 & 0,11 \\
\hline $20: 0$ & - & - & - & 5,48 & 5,39 & 0,06 & - & - & - \\
\hline $22: 0$ & - & - & - & 5,97 & 5,43 & 0,27 & - & - & - \\
\hline
\end{tabular}

1) $n=$ Zahl der Bestimmungen

$\left.{ }^{2}\right)$ Kurzbezeichnung f. Fettsäuren (Zahl der Kohlenstoffatome: Zahl der Doppelbindungen

3) für den Fettsäureanteil

modifizierten Einspritzzyklus (Opt. 024) und $100 \mu$ Injektionsflaschen konnte das bei automatischer Einspritzung erforderliche Mindestvolumen der Probe auf $20 \mu l$ gesenkt werden.

Die Lösung der Fettsäure-2-methoxyäthylester, die nach der Umesterung einer Lipidklasse (Cholesterinester, Triglyceride, Lecithin, Kephalin) aus $1 \mathrm{ml}$ Blut bzw. Plasma anfält, wird auf 20-200 $\mu$ l eingeengt. $1-10 \mu l$ werden automatisch eingespritzt.

\section{GC-Bedingungen}

Säule $2,4 \mathrm{~m} \times 3 \mathrm{~mm}$ i.D. silanisiertes Glas, $100 \mathrm{~g}$ SILAR-10C, bzw. $100 \mathrm{~g}$ OV 275/1 kg 80-100 mesh GAS-CHROM Q Trägergas Helium, $25 \mathrm{ml} / \mathrm{min}$, Einspritzblock $240^{\circ} \mathrm{C}$, Detektor (FID) $260^{\circ} \mathrm{C}$, Ofen $140-240^{\circ} \mathrm{C}$ bzw. $110-230^{\circ} \mathrm{C}$ programmiert (s. Abb. 1, 2)

\section{Ergebnisse und Diskussion}

\section{Umesterung}

Ein Gemisch von insgesamt $0,20 \mathrm{mmol}$ Fettsäuremethylestern wurde in $10 \mathrm{ml} \mathrm{Hexan}$ gelöst. Proben von jeweils $100 \mu \mathrm{l}$ wurden entnommen und die Methylester nach der für unpolare Lipide angegebenen Methode in die 2-Methoxyäthylester überführt. Auf die Extraktion folgte die gaschromatographișche Bestimmung (s. Tab. 1). Die prozentuale Zusammensetzung des Gemisches wurde aus den elektronisch integrierten Peakflächen berechnet. Stoffspezifische Korrekturfaktoren wurden für den Capronsäure- (6:0) und den Önanthsäureester (7:0) verwendet. Für Fettsäuren mit 6-10 Kohlenstoffatomen lag der Variationskoeffizeint (VK) unter $2 \%$, eine Verfälschung des Ergebnisses durch unkontrollierte Verdampfungsverluste ist demnach auszuschließen. Aus dem Anteil der nicht umgeesterten Methylester wurde eine Ausbeute von 98-99\% ermittelt. Bei einem dünnschichtchromatographisch aus Butterfett isolierten Triglyceridgemisch wurde in entsprechender Weise ver- fahren. Der VK für die kurzkettigen Fettsäuren 6:0, $8: 0$ und 10:0 lag unter $3 \%$. Für Buttersäure wurde ein VK von $7,3 \%$ gefunden.

Nach der gleichen Methode erfolgte die Veresterung kurzkettiger Fettsäuren: Einer Lösung von insgesamt $5,5 \mathrm{mmol}$ Carbonsäuren in $20 \mathrm{ml} \mathrm{Chloroform}$ wurden Proben von jeweils $20 \mu$ entnommen und unter Zusatz von $50 \mu$ l Pentadecansäure-Standardlösung (s. Methodik) verestert (s. Tab. 2). Der VK betrug 3-5\%, wobei zu beruicksichtigen ist, daß ein Anteil von etwa $1 \%$ auf den Pipettierfehler entfällt. Die Ergebnisse zeigen, daß mit der Methode geringe Mengen freier und veresterter Fettsäuren zuverlässig erfaßt werden.

Über die Veresterung gesättigter kurzkettiger Fettsäuren mit 2-Chloräthanol haben Oette \& Ahrens (15) berichtet.

Tab. 2. Veresterung kurzkettiger Fettsäuren zu den 2-Methoxyäthylestern

\begin{tabular}{|c|c|c|c|c|c|}
\hline \multirow{2}{*}{ FS } & \multirow{2}{*}{$\begin{array}{l}\text { Einwaage } \\
(\mu \mathrm{mol})\end{array}$} & \multirow{2}{*}{$\begin{array}{l}\left.\text { gefunden }{ }^{1}\right) \\
x(n=5)\end{array}$} & \multirow[b]{2}{*}{ $\pm s$} & \multicolumn{2}{|c|}{$\begin{array}{l}\text { stoffspezifischer } \\
\text { Korrekturfaktor }{ }^{2} \text { ) }\end{array}$} \\
\hline & & & & $x(n=7)$ & $\pm s$ \\
\hline $3: 0$ & 1,34 & 0,78 & 0,03 & 1,705 & 0,010 \\
\hline $4: 0$ & 1,10 & 1,26 & 0,05 & 1,335 & 0,003 \\
\hline $5: 0$ & 0,96 & 0,94 & 0,03 & 1,170 & 0,002 \\
\hline $6: 0$ & 0,80 & 0,81 & 0,03 & 1,092 & 0,003 \\
\hline $8: 0$ & 0,63 & 0,61 & 0,03 & 1,004 & 0,002 \\
\hline 10:0 & 0,65 & 0,66 & 0,02 & 1,000 & - \\
\hline
\end{tabular}

1) mit den angegebenen Korrekturfaktoren korrigierte Werte

$\left.{ }^{2}\right)$ bezogen auf 10:0 
Das Verfahren läßt sich auch auf die Umesterung gesättigter kurz- und langkettiger Fettsäuremethylester übertragen (16). Nach Berichten über Artefaktbildung (16) und schwankende Ausbeuten (17) sowie nach eigenen Umesterungsversuchen mit Methylestern ungesättigter Fettsäuren ist die Eignung der Methode zur Umesterung von Lipiden fraglich.

Die Umesterung von Lipiden im System Bortrifluorid/ Methanol/Benzol haben Morrison \& Smith (18) untersucht. Maximale Reaktionsgeschwindigkeiten wurden bei einem Bortrifluoridgehalt von 0,59-0,74 mol/1 Gemisch gefunden. Ein entsprechendes Maximum des Umsatzes bei niedriger Katalysatorkonzentration wurde von uns im System Bortrifluorid/2-Methoxyäthanol/ Toluol nicht beobachtet. Chlorwasserstoff ist als Katalysator für die Umesterungen mit 2-Methoxyäthanol weniger geeignet: Umsetzungen mit Chlorwasserstoff $1,1-3,3 \mathrm{~mol} / 1 \mathrm{im}$ geschlossenen Rohr bei $60-80^{\circ} \mathrm{C}$ und Reaktionszeiten von $1-2 \mathrm{~h}$ führten zur Bildung von Artefakten. Bei $20^{\circ} \mathrm{C}$ lief die Reaktion zwar einheitlich ab, jedoch wurden Reaktionszeiten von mehreren Stunden benötigt.

2-Methoxyäthylester wurden bisher noch nicht als Derivate der Fettsäuren für die Gaschromatographie eingesetzt. Dünges (19) beschreibt als Derivatisierungsmethode für organische Säuren die Alkylierung mit Chlormethylenmethyläther zu Methoxymethylestern. Die Methode, die für Barbitursäuren entwickelt wurde, befindet sich für Fettsäuren noch im Stadium der Erprobung. Alkylierungs- und Silylierungsreaktionen, die vielfach zu Derivatisierung organischer Säuren herangezogen wurden, sind ausschließlich mit freien Fettsäuren oder deren Salzen, nicht dagegen mit veresterten Fettsäuren möglich. Analy tische Anwendung haben die 2-Methoxyäthylester für die photometrische Bestimmung der Fettsäuren (20) sowie als Substrate zur Enzymbestimmung gefunden $(21,22)$.

Lipide wurden bisher nicht mit 2-Methoxyäthanol umgeestert. Für die Umesterung kurzkettiger Carbonsäureester mit diesem Alkohol finden sich in der Literatur vereinzelt Beispiele: Beránek et al. (23) untersuchten die Kinetik der Umesterung von Essigsäureäthylestern am Ionenaustauscher, Hihara et al. (24) die Umesterung des Methacrylsäuremethylesters in Gegenwart eines Aminsalzes der $p$-Toluolsulfonsäure.

Durch Einsatz des Bortrifluorid-Diäthyläther-Komplexes wird das Arbeiten mit gasförmigem Bortrifluorid vermieden. Das Reagenz wurde bisher nicht als Katalysator für die Umesterung verwendet. Sowa et al. (25) beschreiben die Veresterung einiger aromatischer Carbonsäuren mit diesem Katalysator. Die Synthese wurde in neuerer Zeit von Marshall et al. (26) sowie von Kodaba $(27,28)$ untersucht, der auch Veresterungen mit 2Methoxyäthanol angibt. Sie wurden unter Rückfluß vorgenommen, wobei Reaktionszeiten von mehreren Stun- den erforderlich waren. Bei der von uns angegebenen Methode wird der Diäthyläther aus dem BortrifluoridKomplex durch 2-Methoxyäthanol in einer Austauschreaktion freigesetzt und kann abdestilliert werden. Dadurch ist es möglich, Umesterungen bei höherer Temperatur innerhalb kurzer Zeit quantitativ durchzuführen.

\section{Dünnschichtchromatographie .,}

Die Mengen der für die dünnschichtchromatographischen Trennungen eingesetzten Lipide wurden so gewählt, daß sie annähernd den Lipidmengen aus $1 \mathrm{ml}$ Plasma entsprachen: Es wurde eine Lösung von 0,24 mmol Triglyceriden und $0,33 \mathrm{mmol}$ Cholesterinestern in $10 \mathrm{ml}$ Chloroform hergestellt. Mit jeweils $100 \mu$ dieser Lösung wurde die dünnschichtchromatographische Trennung auf $50 \times 200 \mathrm{~mm}$ Platten durchgeführt, wie im Methodik-Teil für unpolare Lipide beschrieben. Zur Triglyceridfraktion wurden anschließend $100 \mu$, zur Cholesterinesterfraktion $50 \mu \mathrm{l}$ Pentadecansäure-Standardlösung (s. Methodik) hinzugefügt. Nach dem Umestern und der Aufarbeitung erfolgte die Bestimmung der dünnschichtchromatographisch gereinigten Ëster (s. Tab. 1. Abb. 1). Die Ausbeute wurde auf die Menge der umgeesterten Pentadecansäure bezogen. Sie betrug $79,2 \pm 1,6 \%$ für die Triglyceride und $82,0 \pm 1,9 \%$ für die Cholesterinester. Durch Verlängerung der Reaktionszeit auf 30-45 min konnte die Ausbeute auf über 9.0\% gesteigert werden, wodurch sichergestellt war, daß bei der Dünnschichtchromatographie und der Aufarbeitung keine nennenswerten Verluste auftraten. Da man die Bestimmung der unpolaren Lipide mit Tricaprin bzw. Cholesterincaprinat als innerem Standard durchführt (s. Methodik), kann auf eine quantitative Umesterung verzichtet werden.

25,4 $\mu \mathrm{mol}$ Lecithin wurden in Chloroform/Methanol $(5 \mathrm{ml}+5 \mathrm{ml})$ gelöst. Jeweils $1 \mathrm{ml}$ dieser Lösung wurde für die Dünnschichtchromatogrảphie verwendet. Nach Zusatz von $100 \mu l$ Pentadecansäure-Standardlösung (s. o.) wurde nach der für polare Lipide angegebenen.

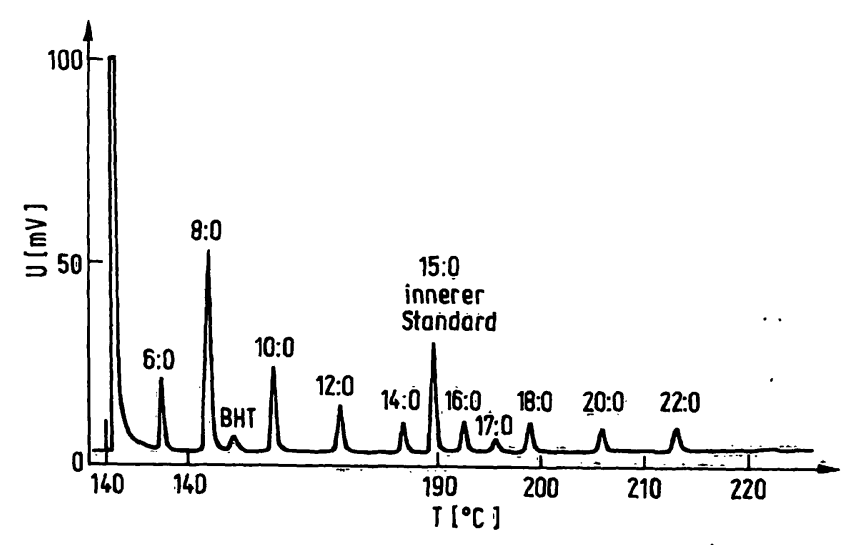

Abb. 1. Gaschromatogramm der 2-Methoxyäthylester, die durch Umesterung eines Gemisches reiner Triglyceride erhalten wurden auf SILAR 10C, Temperaturprogramm: $140^{\circ} \mathrm{C} 8 \mathrm{~min}$ isotherm, $140-190^{\circ} \mathrm{C} 2^{\circ} \mathrm{C} / \mathrm{min}, 190$ $240^{\circ} \mathrm{C} 1{ }^{\circ} \mathrm{C} / \mathrm{min}$. 
Methode umgeestert und aufgearbeitet. Die Bestimmung der Ester erfolgte ohne weitere Reinigung. Die Ausbeute, bezogen auf die Menge der umgeesterten Pentadecansäure, betrug bei fünf Bestimmungen 67,6 $\pm 8,2 \%$. Wird bei der Bestimmung von Lecithin mit Pentadecansäure als innerem Standard ausgewertet, so muß auf vollständigem Umsatz umgerechnet werden. Die Umesterung nach vorausgehender Elution des Lecithins mit Chloroform/Methanol (10 $\mathrm{ml}+10 \mathrm{ml})$ ergab unter sonst gleichen Bedingungen 90\% der Ausbeute. Wird besonders auf die Richtigkeit bei der Absolutbestimmung des Lecithins Wert gelegt, so ist die Elution zweckmäßig, falls man nicht Lecithin mit geeigneter Säurekomponente als inneren Standard einsetzt.

\section{Gaschromatographie}

Um die Analyse von 2-Methoxyäthylestern mit Kettenlängen von 3-24 Kohlenstoffatomen innerhalb einer akzeptablen Zeit durchführen zu können, wählten wir die temperaturprogrammierte Arbeitsweise. Als Trennflüssigkeit wurde SILAR-10C (Cyanopropylsilikon) eingesetzt, das hinsichtlich Selektivität und thermischer Stabilität den Polyesterphasen überlegen ist (29). Als alternative Phase kommt OV-275 in Frage, das ähnliche Eigenschaften besitzt (30). Zur Identifizierung der 2-Methoxyäthylester wurden die relativen Retentionszeiten bei konstanter Temperatur und konstantem Trägergasstrom aus SILAR-10C bestimmt und mit den Werten authentischer Proben (s. Tab. 3) verglichen. Die Vergleichstandards wurden durch die Umesterung von Methyl- oder Äthylesterstandards bzw. durch die Veresterung von Fettsäurestandards (s. Material) erhalten. Die Angabe der äquivalenten Kettenlängen (ECL) erleichtert die Beurteilung der Retentionsdaten. Bei ihrer Berechnung blieben die systematisch abweichenden Werte für die Ester 8:0 bis 11:0 unberücksichtigt.

Der Kontrolle dienten Analysen auf Apiezon L (s. Tab. 3) sowie die katalytische Hydrierung der 2-Methoxyäthylester mit Platin[IV]-oxid, die innerhalb von $20 \mathrm{~min}$ quantitativ verlief.

Stoffspezifische Korrekturfaktoren wurden mit Gemischen der reinen 2-Methoxyäthylester ermittelt. Sie zeigen bis zum Caprylsäureester (8:0) mit zunehmender Kettenlänge einen deutlichen Anstieg (Ta. 2) Für längerkettige Ester wurden annähernd übereinstimmende Korrekturfaktoren gemessen.

Bei der Umesterung der Cholesterinester wird das Cholesterin weitgehend in ein Umwandlungsprodukt, vermutlich Cholesten(5)-yl(3)-(2-methoxyäthyl)-äther, übergeführt, das im Gegensatz zum Cholesterin bei der dünnschichtchromatographischen Reinigung der 2-Methoxyäthylester nicht abgetrennt wird. Im Gaschromatogramm erscheint.es im Anschluß an die Ester. Um Störungen späterer Analysen auszuschließen, muß vor Beginn eines neuen Analysencyclus die Elution des Produktes abgewartet werden.

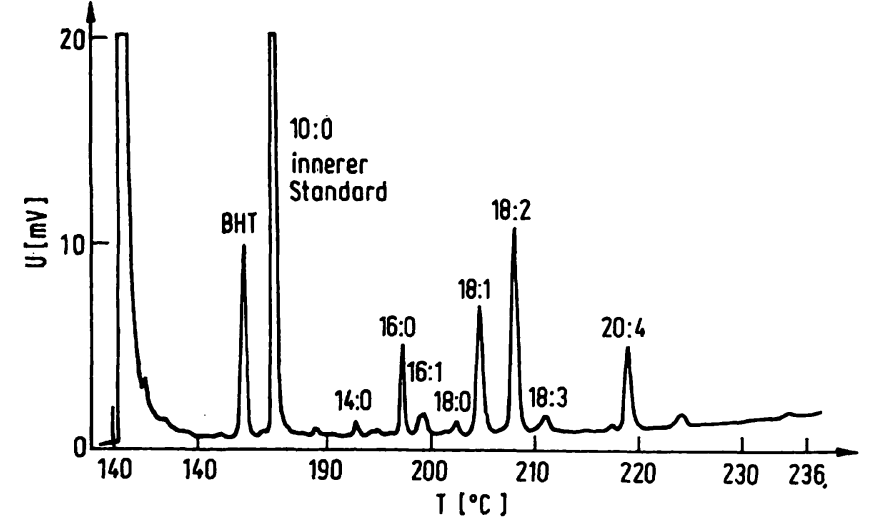

Abb. 2. Gaschromatogramm der 2-Methoxyäthylester, die durch Umesterung der Cholesterinesterfraktion eines Hundeplasmas erhalten wurden auf SILAR-10C, Temperaturprogramm: $140^{\circ} \mathrm{C} 8 \mathrm{~min}$ isotherm, $140-190^{\circ} \mathrm{C} 4^{\circ} \mathrm{C} / \mathrm{min}$, $190-240^{\circ} \mathrm{C} 1{ }^{\circ} \mathrm{C} / \mathrm{min}$.

Tab. 3. Relative Retentionszeiten und Äquivalente Kettenlängen (ECL) der 2-Methoxyäthylester auf gepackten Säulen mit SILAR10C und Apiezon L als stationären Phasen.

\begin{tabular}{|c|c|c|c|c|}
\hline \multirow[b]{2}{*}{$\begin{array}{l}\text { 2-Methoxy-1) } \\
\text { äthylester }\end{array}$} & \multicolumn{2}{|c|}{$\begin{array}{l}\text { Relative } \\
\text { Retentionszeiten }\end{array}$} & \multicolumn{2}{|l|}{ ECL } \\
\hline & $\begin{array}{l}\text { SILAR-2) } \\
\text { 10C }\end{array}$ & $\begin{array}{l}\left.\text { Apiezon }{ }^{3}\right) \\
\text { L }\end{array}$ & $\begin{array}{l}\text { SILAR- } \\
10 \mathrm{C}\end{array}$ & $\begin{array}{l}\text { Apiezon } \\
\text { L }\end{array}$ \\
\hline $\begin{array}{l}8: 0 \\
9: 0 \\
10: 0 \\
11: 0 \\
12: 0 \\
13: 0 \\
14: 0 \\
14: 1(n-5) \\
15: 0 \\
16: 0 \\
16: 1 \operatorname{tr} . \Delta 9 \\
16: 1(n-7) \\
17: 0 \\
18: 0 \\
18: 1 \operatorname{tr} . \Delta 9 \\
18: 1(n-9) \\
18: 2 \operatorname{tr} . \operatorname{tr} . \Delta 9,12 \\
18: 2(n-6) \\
18: 3(n-3) \\
19: 0 \\
20: 0 \\
20: 1(n-15) \\
20: 3(n-6) \\
20: 4(n-6) \\
20: 5(n-3) \\
21: 0 \\
22: 0 \\
22: 1(n-9) \\
22: 6(n-3) \\
23: 0 \\
24: 0 \\
24: 1(n-9) \\
\text { (n) }\end{array}$ & $\begin{array}{l}0,08 \\
0,10 \\
0,13 \\
0,17 \\
0,22 \\
0,29 \\
0,37 \\
0,48 \\
0,47 \\
0,61 \\
0,71 \\
0,75 \\
0,78 \\
1,00 \\
1,13 \\
1,19 \\
1,38 \\
1,55 \\
2,07 \\
1,28 \\
1,63 \\
1,78 \\
2,95 \\
3,24 \\
4,29 \\
2,09 \\
2,67 \\
3,16 \\
7,90 \\
3,42 \\
4,37 \\
5,15\end{array}$ & $\begin{array}{l}0,03 \\
0,04 \\
0,06 \\
0,08 \\
0,12 \\
0,17 \\
0,24 \\
0,23 \\
0,34 \\
0,49 \\
- \\
0,45 \\
0,70 \\
1,00 \\
- \\
0,88 \\
0,85 \\
0,85 \\
1,43 \\
2,02 \\
1,74 \\
1,52 \\
1,37 \\
1,37 \\
2,91 \\
4,13 \\
3,55 \\
2,49 \\
5,85 \\
8,28 \\
7,08\end{array}$ & $\begin{array}{l}- \\
- \\
- \\
- \\
12,00 \\
13,00 \\
14,00 \\
15,04 \\
15,00 \\
16,00 \\
16,59 \\
16,85 \\
17,00 \\
18,00 \\
18,52 \\
18,73 \\
19,31 \\
19,78 \\
20,96 \\
19,00 \\
20,00 \\
20,34 \\
22,40 \\
22,77 \\
23,91 \\
21,00 \\
22,00 \\
22,67 \\
26,37 \\
23,00 \\
24,00 \\
24,66\end{array}$ & $\begin{array}{l}- \\
- \\
- \\
- \\
12,00 \\
13,00 \\
14,00 \\
13,88 \\
15,00 \\
16,00 \\
- \\
15,77 \\
17,00 \\
18,00 \\
- \\
17,64 \\
\overline{17,53} \\
17,53 \\
19,00 \\
20,00 \\
19,58 \\
19,18 \\
18,89 \\
18,89 \\
21,00 \\
22,00 \\
21,55 \\
20,55 \\
23,00 \\
24,00 \\
23,55\end{array}$ \\
\hline Methylester 18:0 & 0,27 & 0,41 & 12,77 & 15,48 \\
\hline
\end{tabular}

1) $14: 1(n-5)=$ cis-Tetradecan (9)-säure, 16:1 tr. $\Delta 9=$ trans-Hexadecen (9)-säure

2) SILAR-10C: $100 \mathrm{~g}$ auf $1 \mathrm{~kg} \mathrm{80-100} \mathrm{mesh} \mathrm{GAS-CHROM} \mathrm{Q}$ Glassäule $2,4 \mathrm{~m} \times 3 \mathrm{~mm}$ i. D., $190^{\circ} \mathrm{C}$, Trägergas - Helium, $25 \mathrm{ml} / \mathrm{min}$.

3) Apiezon L: $100 \mathrm{~g}$ auf $1 \mathrm{~kg} \mathrm{80-100} \mathrm{mesh} \mathrm{Chromosorb} \mathrm{W-AW-}$ DMCS, Glassäulc $1,8 \mathrm{~m} \times 2 \mathrm{~mm}$ i.D., $240^{\circ} \mathrm{C}$, Trägergas Helium, $20 \mathrm{ml} / \mathrm{min}$. 


\section{Beispiel}

Zur Prüfung der Methodik an einer größeren Zahl von Proben wählten wir ein Tierexperiment im Rahmen der laufenden Versuche zur Erforschung der Fettembolie. Einem männlichen Schäferhund-Bastad, $21 \mathrm{~kg}$, wurde in Ketanest-Narkose einseitig in Femurmitte eine Fraktur gesetzt. Nach $6 \mathrm{~h}$ traten Respirationsstörungen auf, exitus letalis trat nach etwa $16 \mathrm{~h}$ ein. Der histologische Lungenbefund einer mittelschweren Fettembolie wurde durch die gaschromatographische Bestimmung der Triglyceride im Lungengewebe bestätigt, die einen Gehalt von $17 \mathrm{mmol} / \mathrm{kg}$ Trockensubstanz ergab. Die Blutentnahme erfolgte jeweils nach zwölfstündigem Fasten arteriell und zentralvenös mittels zweier durch die großen Halsgefäße eingeführter Katheter. Es wurden in einem Zeitraum von $48 \mathrm{~h}$ vor und $6 \mathrm{~h}$ nach Setzen der Fraktur Blutproben entnommen. Ein Beispiel für die Fettsäurebestimmungen nach Umesterung der getrennten Lipidklassen ist in Abbildung 3 wiedergegeben. Es wurde mit innerem Standard sowie nach der 100\% Methode ausgewertet. Die Umrechnung der FettsäureWerte in die Lipid-Werte erfolgte unter Berücksichtigung der Zahl der Fetțsäurereste im Lipidmolekül. Die Auswertung nach der $100 \%$-Methode ergibt bei durchschnittlich zehn Bestimmungen für die Hauptbestandteile einen VK von maximal 5\%, während für die Nebenbestandteile mit einem VK bis zu $10 \%$ zu rechnen ist. Wird mit innerem Standard ausgewertet, so betragen die entsprechenden Richtwerte 10\% bzw. 15\%. Für die Summen der Bestandteile beträgt der VK etwa 10\%. Das Auftreten größerer Mengen kurzkettiger Fettsäuren im Blut und in der Lunge wurde nicht beobachtet.

Bei alleiniger Bestimmung der Triglyceride im Plasma hätte nicht ausgeschlossen werden können, daß die nach der Fraktur zu beobachtende Abnahme der Triglyceridfraktion auf einer Abscheidung von PlasmaTriglyceriden beim Zentrifugieren beruhte. Über die gemeinsame Abscheidung von Triglyceriden und Erythrocyten aus dem Blut von Hunden mit beidseitiger Femurfraktur haben Bergentz et al. (31) berichtet. Ausgehend von Citrat-Blut wurde von den Autoren die Bestimmung der Triglyceride im Plasma und in der Erythrocytenmasse durchgeführt. Die Ergebnisse fanden durch Triglyceridbestimmungen im Voll-Blut (32) keine Bestätigung. Eine zuverlässige Beurteilung von Entmischungsvorgängen beim Zentrifugieren des Blutes ist

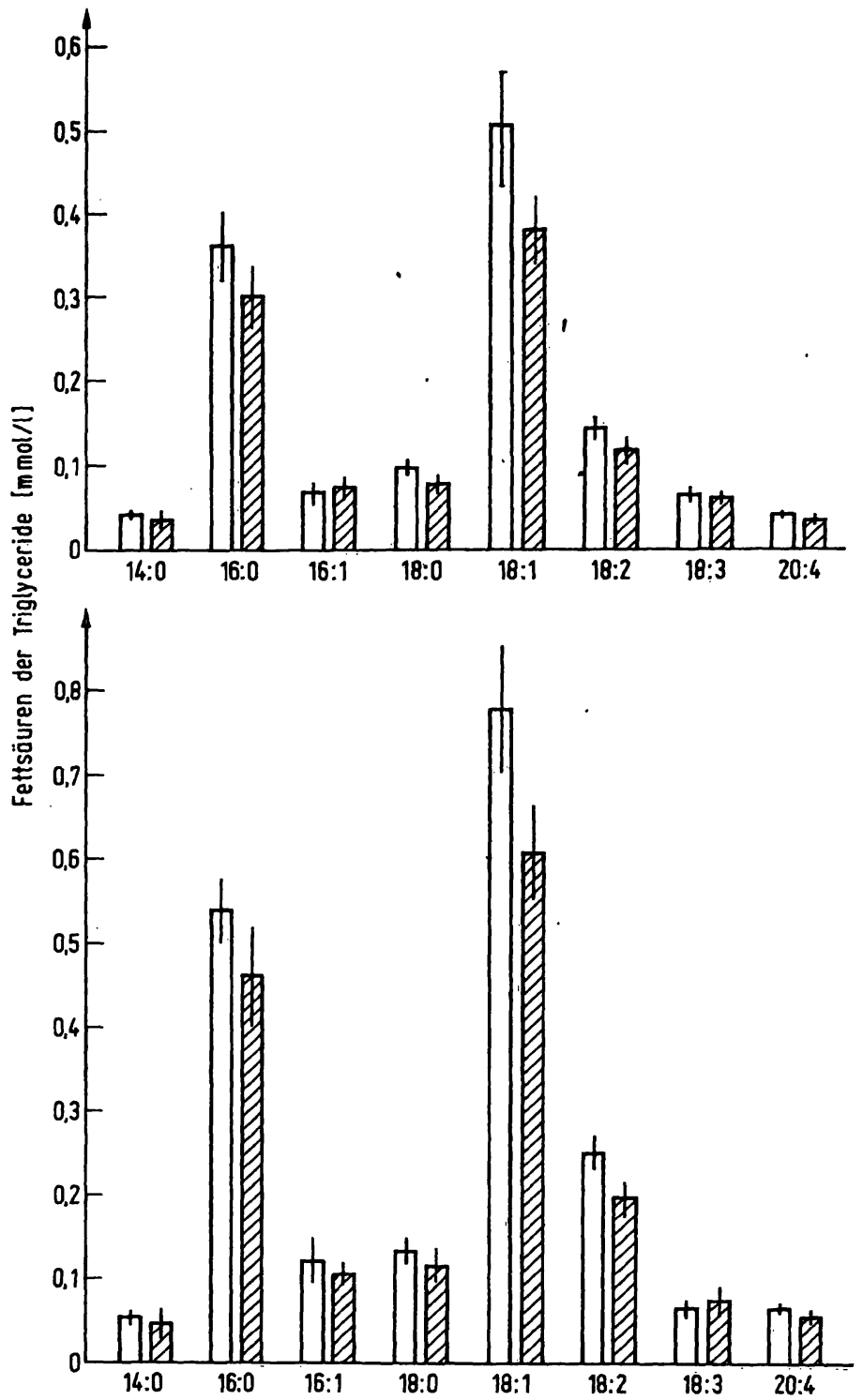

Abb. 3. Fettsäuren der Triglyceride im Blut (oben) und Plasma (unten) eines Hundes $0-48 \mathrm{~h}$ vor Fraktur (weiße Säulen, $\mathrm{n}=3$ für Blut, $\mathrm{n}=8$ für Plasma) und $0-6 \mathrm{~h}$ nach Fraktur (schraffierte Säulen, $n=13$ für Blut, $n=16$ für Plasma).

jedoch erst bei gleichzeitiger Bestimmung der Lipide im Blut und Plasma möglich. Im vorliegenden Fall beweist die parallele Abnahme des Triglyceridgehaltes, daß beim Zentrifugieren keine Entmischung der Plasma-Triglyceride stattfand.

\section{Literatur}

1. James, A. T., Lovelock, J. E., Webb, J. \& Trotter, W. R. (1957), Lancet $I, 705-708$.

2. Tuna, N., Reckers, L. \& Frantz, I. D. jr. (1958), J. Clin. Invest. 37, 1153-1165.

3. Ahrens jr., E. H., Insull jr., W., Hirsch, J., Stoffel, W., Peterson, M. L., Farquhar, J. W., Miller, T. \& Thomasson, H. J. (1959), Lancet $I, 115-119$.

4. Hallgren, B., Stenhagen, S., Svanborg, A. \& Svennerholm, L.
(1960), J. Clin. Invest. 39, 1424-1434.

5. Gnauck, G., Stolz, P., Honigmann, G. \& Singer, P. (1973), Z. Med. Labortechn. 14, 15-23.

6. Vorbeck, M. L., Mattick, L. R., Lee, F. A. \& Pederson, C. S. (1961), Anal. Chem. 33, 1512-1514.

7. Oette, K., Doss, M. \& Winterfeld, M. (1970), diese Z. 8, 525-528.

8. Smith, L. M. \& Hardjo, S. (1974), Lipids 9, 674-678. 
9. Stolz, C. (1971), in Die posttraumatische Fettembolie (Koskowski, L., Heller, W. \& Durst, J.), S. 57, F. K. Schattauer-Verlag, Stuttgart-New York.

10. Heller, W. \& Durst, J. (1971), in l.c. (9) S. 105, 120.

11. Palomaa, M. H. (1909), Chem. Ber. 42, 3873-3878.

12. Farquhar, J. W. (1962), Biochim. Biophys. Acta 60, 80-89.

13. Folch, J., Lees, M. \& Stanley, G. H. S. (1957), J. Biol. Chem. 226, 497-509.

14. Nutter, L. J. \& Privett, O. S. (1968), J. Chromatogr. 35, 519-525.

15. Oette, K. \& Ahrens, E. H., jr. (1961), Anal. Chem. 33, 1847-1851.

16. Karmen, A. J. (1967), J. Lipid. Res. 8, 234-238.

17. Mráz, M. \& Šedivec, U. (1973), Collect. Czech. Chem. Commun. 38, 3426-3433.

18. Morrison, W. R. \& Smith, L. M. (1964), J. Lipid Res. 5, 600-608.

19. Dünges, W. (1973), Chromatographia 6, 196-197.

20. Pilz, W. (1959), Z. Anal. Chem. 166, 189-193.

21. Hanson, H. \& Lasch, J. (1967), Hoppe Seyler's Z. Physiol. Chem. 348, 1525-1539.
22. Brockerhoff, H. (1969), Arch. Biochem. Biophys. 134, 366-371.

23. Beránek, L., K. Setínek \& Kraus, M. (1972), Collect. Czech. Chem. Commun. 37, 2265-2268.

24. Hihara, T., Takizawa, F., Kimeda, T. \& Shiowaki, K. (1973), Jap. Pat. 7348, 412, zit. nach Chem. Abstr. 79, 125881 a.

25. Sowa, F. J. \& Nieuwland, J. A. (1936), J. Amer. Chem. Soc. 58, 271-272.

26. Marshall, J. L., Erickson, K. C. \& Folsom, T. K. (1970), Tetrahedron Lett. 4011-4012.

27. Kodaba, P. K. (1971), Synthesis, 316-317.

28. Kodaba, P. K. (1972), Synthesis, 628-630.

29. Ettre, L. S. (1974), Chromatographia 7, 261-268, 407-415.

30. Gas-Chrom Newsletter (1974), 15, 1.

31. Bergentz, S. E., Gelin, L. E., Hallgren, B., Rudenstam, C. M. \& Svanborg, A. (1962), Acta Chir. Scand. 124, 377385.

32. Burkhardt, K. (1972), Med. Welt 22, 616-620. Prof. Dr. J. D. Kruse-Jarres 78 Freiburg/Brsg. Hugstetterstr. 55 
, 。 